\title{
成年雄性斑胸草雀前脑与中脑对习得性发声控制的侧别差异
}

曾贤燕，李东风*

华南师范大学 生命科学学院, 广东省高等学校生态与环境科学重点实验室, 广州 510631

摘要: 成年雄性鸣禽的习得性发声信号一一长鸣(long call)和鸣唱(song)是由前脑高级发声中枢启动, 以及由前脑最后一级 输出核团弓状皮质栎核(robust nucleus of the arcopallium, RA)整合输出。RA 投射神经元与位于中脑的基本发声中枢丘间 复合体背内侧核(dorsomedial nucleus of the intercollicular, DM)形成突触连接。该文采用电损毁与声谱分析相结合的方法, 通过依次损毁成年雄性玨胸草雀(Taeniopygia guttata)单侧 RA 和 DM 核团, 探讨了前脑和中脑对习得性发声的影响。 结果提示, RA 核团与 DM 核团共同参与了对雄性斑胸草雀习得性声音的调控，而且这种控制具有右侧优势。

关键词: 成年雄性玟胸草雀; 弓状皮质栎核; 丘间复合体背内侧核; 习得性发声; 声谱分析

中图分类号: Q959.739; Q62; Q42 文献标志码: A 文章编号: 0254-5853-(2013)01-0001-07

\section{Lateral differences in the forebrain and midbrain control of learned vocalizations in adult male Zebra Finch (Taeniopygia guttata)}

\author{
Xian-Yan ZENG, Dong-Feng LI * \\ School of Life Sciences, South China Normal University, Key Laboratory of Ecology and Environmental Science in Higher \\ Education of Guangdong Province, Guangzhou 510631, China
}

\begin{abstract}
Learned vocalizations (long call and song) of adult male songbirds start from the high vocal center (HVC), and are integrated and output by the robust nucleus of the arcopallium (RA), which connects synaptic relationships with the dorsomedial nucleus of the intercollicular complex (DM). To determine the effect on learned vocalization of the unilateral forebrain and midbrain in adult male zebra finch, electrolytic lesions and acoustic analysis technology were used. The results indicated that RA and DM nuclei are involved in the control of learned vocalization, and the right side is dominant in the forebrain and midbrain.
\end{abstract}

Keywords: Adult male zebra finch; Robust nucleus of the arcopallium; Dorsomedial nucleus of the intercollicular; Learned vocalization; Acoustic analysis

成年雄性斑胸草雀的习得性发声包括长鸣和 鸣唱，长鸣具有性别二态性，鸣唱则为雄性特有 (Simpson \& Vicario, 1990), 两者都是由前脑高级发 声中枢控制产生。

雄鸟在幼龄期通过模仿和听觉反馈获得习得 性发声 (Brainard \& Doupe，2002; Funabiki \& Funabiki, 2009)。鸣禽脑内离散核团和它们之间的连 接形成了发声神经控制系统，与非鸣禽不同的是， 其前脑内有较多且功能复杂的发声核团。习得性发 声信号由前脑高级发声中枢 (high vocal center, $\mathrm{HVC}$ ) 启动，随后或直接下行至前脑的弓状皮质栋 核(robust nucleus of the arcopallium, RA), 此通路称 发声运动通路(vocal motor pathway, VMP); 或途经 前端脑环路(其中包括基底神经节的 X 区 “X area” 丘脑背外侧核 “medial portion of the dorsolateral nucleus, DLM” 和新纹状体前部巨细胞核外侧部 “ lateral magnocellular nucleus of the anterior neostriatum, LMAN”, 后再行至 RA，此通路称前端 脑通路(anterior forebrain pathway, AFP)(Doupe et al, 2005; Nottebohm, 2005; Doupe et al, 2004; Troyer \& Bottjer, 2001)。RA 作为发声控制通路中前脑的最后 一级输出核团, 其整合了来自 HVC 和前端脑环路 的控制信号，继续发出指令引导下游核团完成发声 控制，是发声控制系统中高级中枢与低级中枢的分

收稿日期: 2012-09-03; 接受日期: 2012-10-10

基金项目：国家自然科学基金资助项目(30970363, 31172092)

*通信作者 (Corresponding author), E-mail: dfliswx@126.com 
界点。研究发现, 雌性鸣禽的 RA 核团极度萎缩 (Nottebohm \& Arnold, 1976; Li et al, 1992)且不与中 脑基本发声中枢形成突触连接(Fukushima \& Aoki, 2002), 以致雌性鸣禽不善唱; 雄性鸣离的 RA 核团 的体积为雌性的数倍, 若损毁两侧 RA 则令雄性长 鸣丧失习得性成分, 与雌鸟的长鸣相似, 这足以说 明 RA 在雄性鸣禽习得性发声中的重要作用。 Vicario et al (2001)报道, 雄性特异性的长鸣需要有 完整的 RA 结构, 当两侧 RA 被损后, 雄鸟的长鸣不 仅失去原来的特征, 其对声音刺激的反应也与正常 雄鸟不同，而与雌鸟的类似，所以 Vicario et al (2001)认为雄鸟的 RA 核团是控制发声行为的性别 差异性的关键核团。丘间复合体背内侧核 (dorsomedial nucleus of the intercollicular complex, DM)位于中脑丘间核(nucleus intercollicularis，Ico) 内，是鸟类最基本的发声中枢 (Gurney，1980; Kojima \& Aoki, 2003), 可以不依赖于前脑直接控制 简单发声, 在雄性鸣禽中, DM 核团接受 RA 投射神 经元的信息输入, 是习得性发声信号位于中脑的重 要中间站, 其发出神经投射至位于延髓的舌下神经 气管鸣管亚核(nXIIts), 完成发声控制。Fukushima \& Aoki (2002)报道, 刺激两性鸣禽 DM 核团能诱发出 类似长鸣的鸣叫, 鸣叫的时程变化与刺激的脉冲数 相关, 鸣叫的频率变化 (只有雄鸟才有)与刺激的脉 冲频率有关, 因此, 雄鸟 DM 由于接受了来自 RA 的突触输入也是个具有性别差异的发声核团。

本研究为探讨成年雄性鸣禽前脑与中脑对习 得性发声的影响, 采用电损毁与声谱分析相结合的 方法, 依次电损毁成年雄性斑胸草雀单侧 RA 与 DM 核团并采集两次手术前后的长鸣和鸣唱。使用 声音分析软件对收集到的长鸣和鸣曲进行处理和 分析, 通过分析习得性发声的变异情况来推测核团 的控声机理。单侧损毁控声核团的方法既能分析脑 内发声信号控制模式又避免了动物失音。

\section{1 材料方法}

选用健康成年雄性斑胸草雀 (Taeniopygia guttata) 25 只, 年龄 $>90 \mathrm{~d}$, 体重 $12 \sim 15 \mathrm{~g}$ 。购于广 州花地湾花鸟虫鱼市场, 并在华南师范大学生命科 学学院动物房饲养。实验动物使用符合国家和学校 动物伦理委员会规定并遵守国际惯例。

\section{1 实验流程}

(1) 采集正常状态下的成年雄性习得性声音 (pre-operation, Pre); (2) 第一次损毁手术，电损毁单 侧 RA 核团; (3) 1-2 天后采集第一次术后声音 (post- operation 1, Post 1; (4) 间隔 5-7 天，第二次 损毁手术电损毁同侧 DM核团; (5) 1-2天后采集第 二次术后声音(post-operation 2, Post2); (6) 组织学 检验。

\section{2 声音采集及分析}

将单只雄鸟置于测听室内, 定向话筒靠近笼壁, 距离鸟 $25 \mathrm{~cm}$, 每天录音 $1.5 \mathrm{~h}$, 连续录音 $3 \mathrm{~d}$, 采 样频率为 $44100 \mathrm{~Hz}$, 单声道, 16 位分辨率, 取得正 常状态下、手术后的声音样本, 数字化信息保存为 *.wav 文件。使用 Cool Edit 2000 软件对鸟鸣进行录 制和篮选，读取高端频率 (high frequency, HF); Wavesurfer 声音分析软件绘制语图、波形图, 读出 基频(fundamental frequency, FF)(Li \& Geng, 2005); SAP 声音分析软件(Version1.02)Explore \& Score 界 面下进行语图分析, 读出时程(duration)、音高 (pitch)、调频 (frequency modulation, FM) 及振幅 (amplitude)等值。此外, SAP 软件支持声音比对分析, 即将两组声音输入该软件能得出这两组声音在形 态结构等综合因素上的相似值(Tchernichovski et al, 2000)。采集的声谱图、时间波形图等用 Photoshop 软件 (Version7.0.1) 排版作图。利用 SPSS 软件 (Version 11.5)进行组内单因素方差分析(ANOVA)。 数据用均值 \pm 标准差 $($ mean $\pm S D)$ 表示。 $t$-test 计算差异 显著性, $P<0.05$ 显著性差异, $P<0.01$ 差异极显著。利 用 Origin 软件(Version 8.0)绘制统计图。

\section{3 脑内核团电损毁}

动物麻醉，胸大肌注射 $0.03 \mathrm{~mL} 10 \%$ 水合氯 醛, 数分钟后动物进入麻醉状态, 剪去头顶及耳孔 处毛。在立体定位仪上固定头部，两耳杆伸入耳孔 抵着耳前骨，嚎杆上固定嗄，使头部向下倾斜 $45^{\circ}$, 头部固定完毕。剪开头皮暴露顿骨, 可见人字缝, 将人字缝交点处作为零点, 依据斑胸草雀脑图谱 (Nixdorf-Bergweiler \& Bischof, 2007)定位核团, 用 尖头镊子在颅骨上开直径 $1 \mathrm{~mm}$ 小孔，揭开脑膜 后, 自电极尖端接触脑表面算起, 下电极至坐标深 度, 打开刺激器开关, 先调至脉冲输出模式, 后调 至恒流电模式 $1 \mathrm{~mA}, 10 \mathrm{~s}$, 完成损毁, 抽离电极, 止 血封闭伤口。

\section{4 灌流取脑制片}

动物麻醉, 胸大肌注射 $~ 0.05 \mathrm{~mL} 10 \%$ 水合氯 醛; 仰卧固定于手术板, 剪去颈部毛, 剪开颈部皮 
肤，暴露颈总动脉，分离颈部两侧大静脉。先用 $0.75 \%$ 生理盐水颈动脉灌注 $5 \mathrm{~min}$, 再换 $4 \%$ 多聚甲 醛固定液灌注约 $30 \mathrm{~min}$, 剥去硕骨取全脑浸泡在多 聚甲醛固定液中固定 $24 \mathrm{~h}$ ，换 30\%蔗糖溶液浸泡至 标本沉底。使用冰冻切片机将鸟脑冠状切片, 片厚 $40 \mu \mathrm{m}, \mathrm{Nissl}$ 染色后中性树脂封片，镜检。

\section{2 结 果}

\section{1 损毁右侧核团对雄性长鸣造成显著影响}

正常状态下雄性斑胸草雀长鸣频率范围

500 20 000 Hz，基频稳定，谐波清晰，最强能量带 在 $3000 \sim 6000 \mathrm{~Hz}$ 之间; 富含快速调频特性，即声 音频率在极短时间内(15 50 ms)快速上升，后缓慢 下降，声音婉转动听(图 1 左)。该声音在地面可传播 $100 \mathrm{~m}$, 故亦称长距离鸣叫(distance call)。

损毁右侧 RA 核团后，长鸣失去快速调频特性， 谐波变得平缓，谐波条纹模糊; 主能量带宽扩大，
界线不清; 时程出现显著延长(图 1 中)。将音频数 字化后分析，发现各声学参数与损毁前相对比均出 现了显著差异：鸣叫时程由 $165.39 \mathrm{~ms}(\bar{x})$ 延长至 $184.1927 \mathrm{~ms}(\bar{x})$, 鸣叫时程延长约 1.1 倍; 音高(音

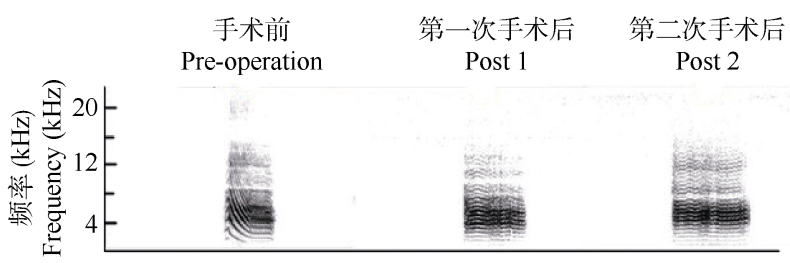

图 1 右侧实验组 3 个阶段长鸣语图

Figure 1 Sonogram of male's long call of right side experimental group at three stages

左图: 对照; 中图: 损毁右侧 RA 后的长鸣; 右图: 再损毁右侧 DM 后的长鸣。

Left: control; Middle: right side RA lesion; Right: right side DM lesion following RA lesion.
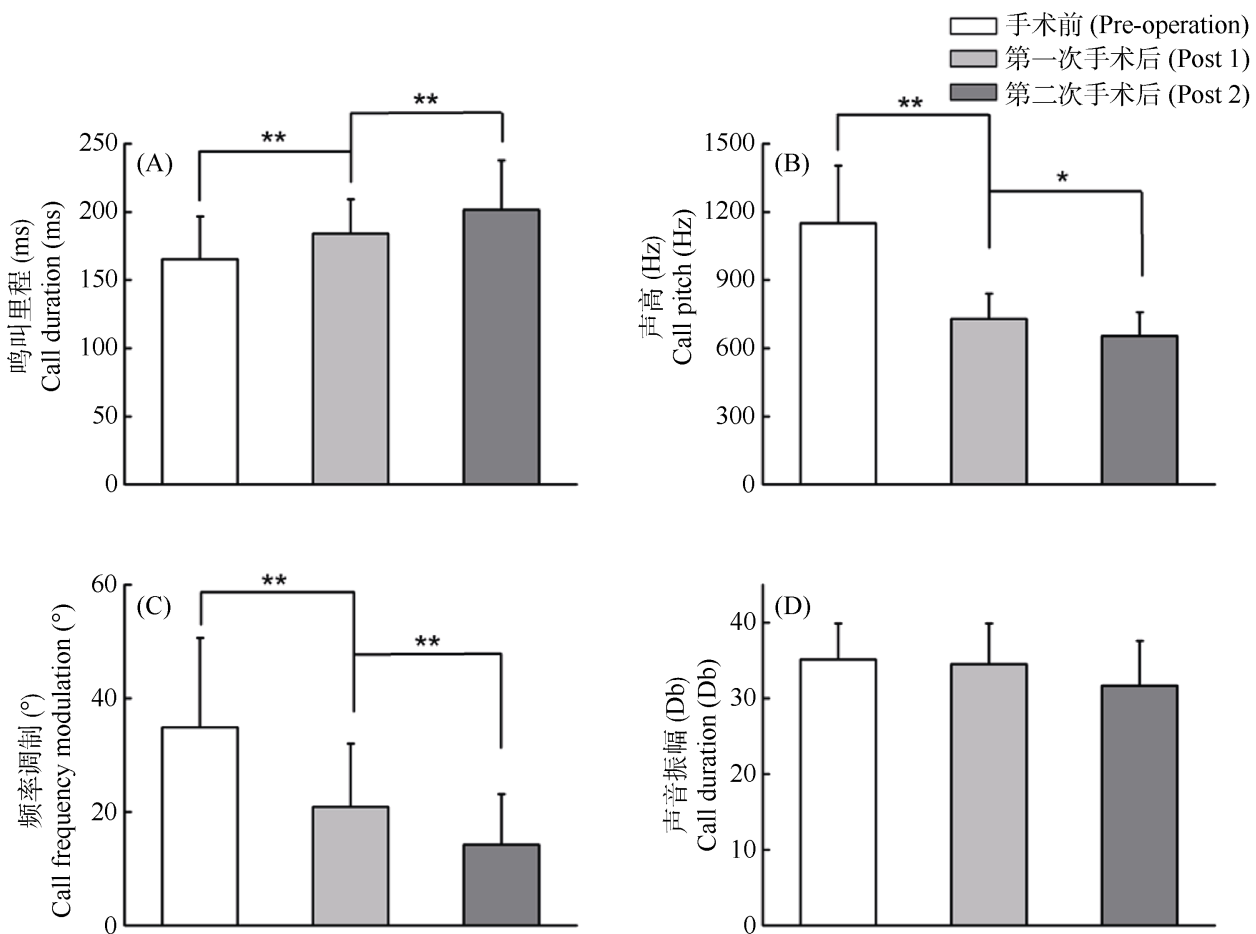

图 2 右侧损毁组 3 个阶段长鸣的量化分析 ( $n=5$ 例)

Figure 2 Quantification of long call in adult male zebra finches $(n=5)$ at three stages with lesions on right side $\operatorname{Pre}($ 白): 手术前; Post1(浅灰): 第一次手术后; Post2(深灰): 第二次手术后。图 A: 手术前后鸣叫时程的差异, 右侧 RA 损毁后鸣叫时程延长了 1.1 倍，而接着右侧 $\mathrm{DM}$ 损毁后鸣叫时程又延长了 0.9 倍，两次手术共使长鸣时程延长了 1.2 倍。图 $\mathrm{B}$ : 手术前后鸣叫音高的差异，右侧 $\mathrm{RA}$ 损毁后，长鸣 音高下降了 $37 \%$, 但 DM 损毁后音高没有再出现明显变化。图 C: 手术前后调频的差异, RA 损毁后调频值下降了 $40 \%$, 接着 DM 损毁后该值再次下 降了 $31.9 \%$ 。图 D: 手术前后振幅的变化存在个体差异性。

Pre: preoperation (white), Post1: postoperation1(light gray), Post2: postoperation2 (dark gray). A: Differences in call duration before and after operations. 1.1 times elongation after RA was destroyed and 0.9 times elongation after DM was destroyed, the total elongation of both operations was 1.2 times compared with the control. B: Differences in pitch before and after operations. After RA was destroyed, long call pitch decreased 37\%, but no more changes were observed after DM was destroyed. C: Differences in frequency modulation before and after operations. After RA was destroyed, the FM value decreased by $40 \%$, with a further 31.9\% decrease after DM was destroyed. D: Changes in amplitude before and after operation showed individual differences. 
调) 由 $1151.19 \mathrm{~Hz}(\bar{x})$ 降至 $728.5138 \mathrm{~Hz}(\bar{x})$, 降 幅为 $37 \%$, 声色由高光变低平; FM 值 (调频指数) 由 $34.93(\bar{x})$ 变至 $20.9174(\bar{x})$, 降幅为 $40 \%$, 叫声不 再婉转; 振幅无明显变化, 有个体差异性(图 2)。此 时的长鸣已不具有雄性长鸣的特征, 与雌性非习得 性长鸣在时域和频域上都十分相似。

$\mathrm{RA}$ 损毁后 1 2 d 动物已恢复常态, 采集足够 声音信号, 5 7 d 后进行同侧 DM 核团的损毁。两 次术后, 从语图上观长鸣的结构与第一次术后的相 比无明显差异(图 1 右)。继续发生变化的声学参数 有: 长鸣时程, 此时长达 $201.44 \mathrm{~ms}(\bar{x})$, 长鸣时程 再次显著延长 $9 \%$, 两次手术共使雄鸟的长鸣延长 了 1.2 倍; 音高降为 $653 \mathrm{~Hz}(\bar{x})$, 与第一次术手相比 下降 $10 \%$, 谐波模糊, 音色暗哑; FM 值降为 $14.2472(\bar{x})$, 第二次术后再次下降 31.9\%, 声音趋 于恒频(图 2)。

损毁右侧 RA 核团后长鸣的声学结构与正常时 的相比相似度仅为 $67 \%$, 进而右侧 $\mathrm{DM}$ 核团缺失后 相似度与正常相比不到 $60 \%$, 但与第一术后相比没 有明显差异, 即第二术后叫声结构没有再发生显著 变化(图 3)。

\section{2 损毁左侧核团未对雄性长鸣造成影响}

图 4 为左侧损毁组语图。图中由左到右分别是

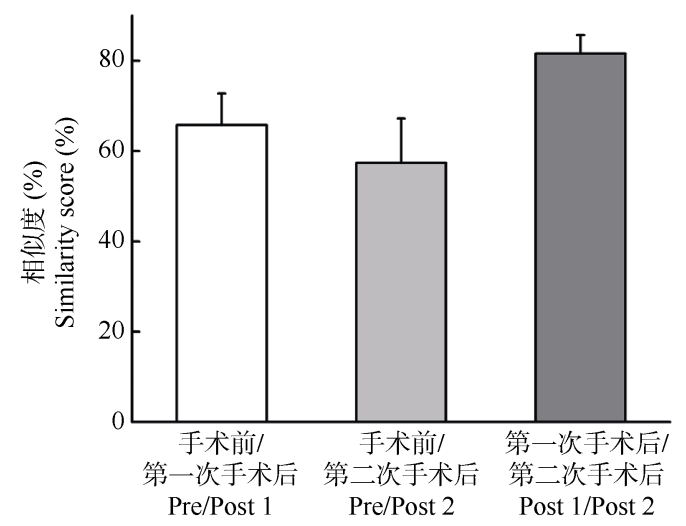

图 3 右侧损毁组手术前后长鸣结构的相似度比较

Figure 3 Similarity score of long call construction before and after operations with lesions on right side

Pre/Post1(白): 右侧 RA 损毁后长鸣的结构与正常相比仅有 $67 \%$ 的相似度; Pre/Post2(浅灰): 右侧 DM 损毁后长鸣的结构与正常相比亦只有较低的 相似性; Post1/Post2(深灰): 第一次术后与第二次术后长鸣结构相似度 较高说明第二次损毁并未影响声学结构。

Pre/Post1 (white): call construction after right side RA was destroyed had only $67 \%$ similarity compared with control. Pre/Post2 (light gray): low similarity compared with control after right side DM was destroyed. Post1/Post2 (dark gray): similarity of Post1 and Post2 was high, indicating that the second lesion operation did not affect the acoustic construction.

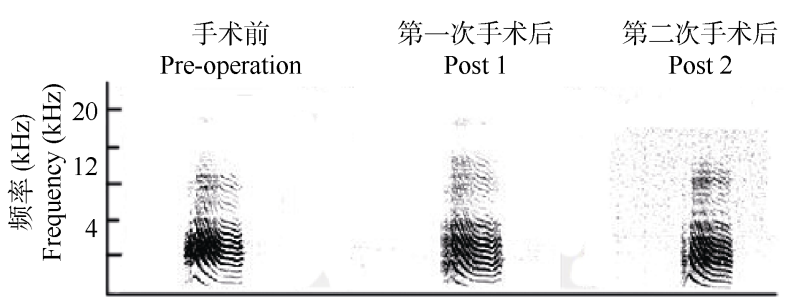

图 4 左侧实验组长鸣语图

Figure 4 Sonogram of male's long call of left side experimental group at three stages

左图：对照; 中图: 损毁左侧 RA 后的长鸣; 右图: 再损毁左侧 DM 的长鸣。

Left: control; Middle: left side RA lesion; Right: left side DM lesion following RA lesion.

术前正常状态下的雄性长鸣、左侧 RA 核团电损毁 后的长鸣、左侧 $\mathrm{DM}$ 核团电损毁后的长鸣。手术前 后的声音均未呈现明显的变化, 无论是从谐波的走 向、能量带的分布还是时程上的特征。将各声学参 数提取出来进行组内单因素方差分析, 如表 1 所示, 长鸣的时程、音高(音调)、FM、振幅 (响度) 在术 前术后 3 个阶段均未出现显著性差异 $(P>0.05)$ 。

表 1 左侧实验组 3 个阶段长鸣的声学参数

Table 1 Acoustic parameters of left side experimental group's long calls in three stages

\begin{tabular}{lllll}
\hline & Duration (ms) & Pitch $(\mathrm{Hz})$ & $\begin{array}{l}\text { Frequency } \\
\text { modulation }(\mathrm{deg})\end{array}$ & Amplitude (Db) \\
\hline Pre & $164.41 \pm 22.95$ & $997.83 \pm 402.98$ & $40.33 \pm 20.59$ & $37.50 \pm 4.66$ \\
P1 & $154.98 \pm 27.20$ & $769.43 \pm 132.75$ & $34.58 \pm 18.78$ & $33.85 \pm 6.52$ \\
P2 & $151.98 \pm 36.13$ & $859.43 \pm 252.51$ & $38.71 \pm 23.48$ & $33.27 \pm 4.21$ \\
$P$ & $>0.05$ & $>0.05$ & $>0.05$ & $>0.05$ \\
\hline
\end{tabular}

损毁左侧核团不会对长鸣结构与时程造成影响, 但音高和调频在 RA 损 毁后出现轻微失稳, 而在 DM 损毁后又恢复。数值 $=$ 平均值 \pm 标准差。

Lesion on side nuclei affected neither construction nor duration, but both pitch and frequency modulation appeared slightly destabilized after RA lesion and recovered after DM lesion. Value $=$ mean $\pm S D$.

\section{3 右侧核团损毁造成鸣曲音节改变}

鸣唱是雄性鸣昺特有的习得性发声, 鸣曲中富 含着习得性的社交意义。鸣曲是成串的声音信号, 它由一至多个序幕音(i)引出的若干串由相同或不 同音节 $(s)$ 组成的短句构成。

分侧损毁结果发现右侧手术组的鸣曲出现显 著变化，而左侧则不明显。图 5 所显示的是同一只 进行右侧损毁的成年雄性斑胸草雀术前术后 3 个阶 段的曲鸣差异。图 5 的上图为对照鸣曲; 中图为 


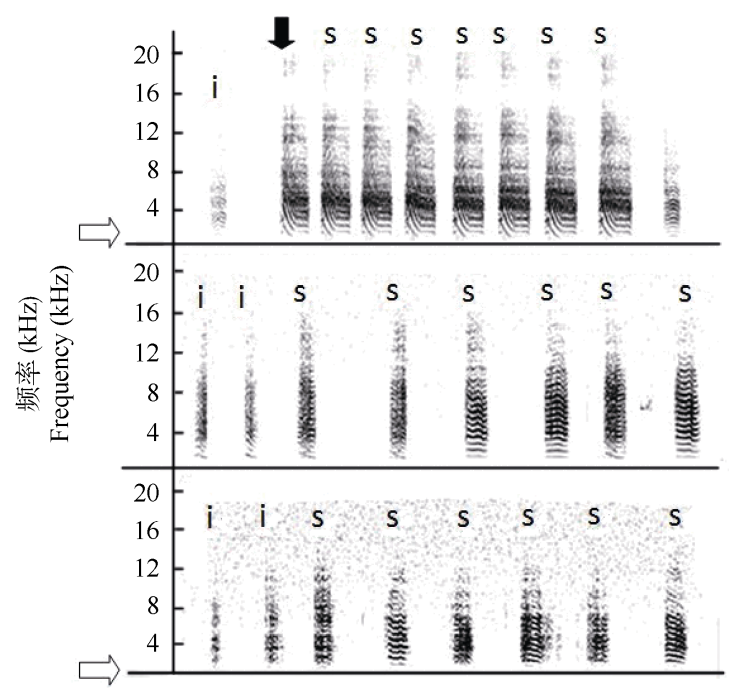

图 5 右侧实验组鸣曲语图

Figure 5 Sonogram of lesioned right side experimental group in three stages

上图: 对照; 中图: 右侧 RA 损毁后鸣曲; 下图: 右侧 DM 损毁后鸣曲(黑 色箭头指示快速调频特性，空心箭头指向基频)。

“i” 表示序幕音; “s” 表示音节。

Top: Control song; Middle: Song with right side RA lesion; Bottom: Song with right side DM lesion with prior ablation of ipsilateral RA (black arrow indicates fast frequency modulation, hollow arrows direct to the fundamental frequency.). i: introductory note; s: syllable.

右侧 RA 损毁后的变形鸣曲; 下图为右侧 DM 损毁 后的变异鸣曲。正常状态下雄性的自鸣曲的序幕音 (i) 一 多个, 相同或不同的音节(s)尾随其后成串 地出现，每个音节间距紧密而稳定，相同的音节时 程也相对固定，单个音节内富含复杂而有规律的频 率调制，基频和高频都十分稳定，这些都是成熟鸣 曲所具有的特征。损毁右侧 RA 核团后，仍然可见 成夰的声音信号发出, 即鸣曲的序列没有受影响, 但鸣曲内的音节出现较大变化, 表现为时程显著缩 短; 结构由原本复杂且有调频变成简单得近似于恒 频，且谐波模糊; 主能量带拓宽且界限不清晰。对 鸣曲中音节的基频和高频进行分析, 发现当右侧 $\mathrm{RA}$ 损毁后基频失去稳定，当右侧 $\mathrm{DM}$ 损毁后基频 出现上移, 幅度为 $66 \%$ (图 6A); 相反, 当右侧 RA 损 毁后，音节频率高端缺失 $22 \%$, 由原来的 $(20$ 282.82 \pm 261.56$) \mathrm{Hz}$ 降至(15 859.67 \pm 402.52$) \mathrm{Hz}$, 继 RA 后损毁右侧 DM 核团, 音节频率高端再次下降 12\%，降至(13 993.81 \pm 1017.67$) \mathrm{Hz}$ ，两次手术后音 节频率高端降幅达 34\%(图 6B)。

\section{4 组织学检验}

采集完每个阶段的声音信号后(约两周), 所有
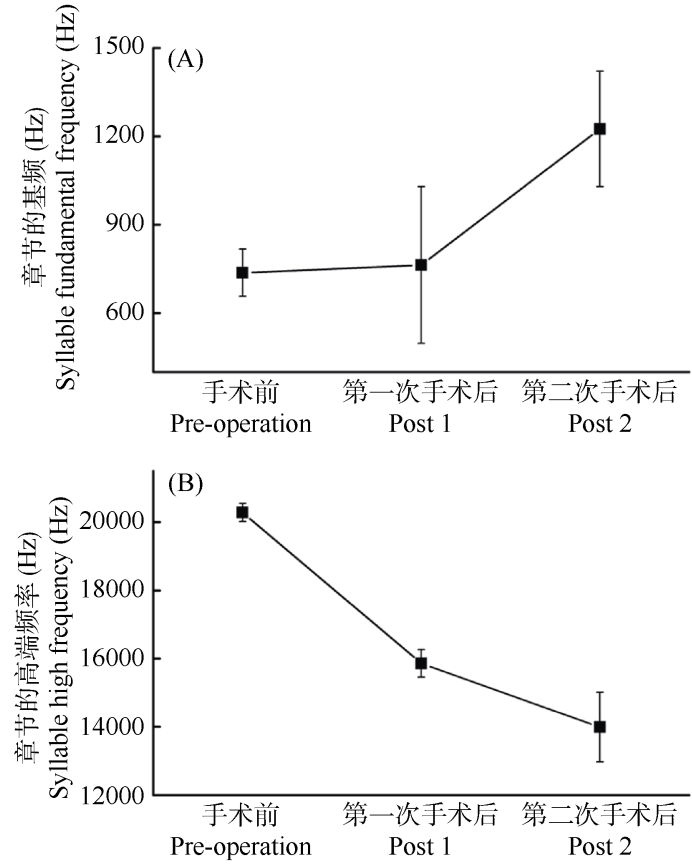

图 6 右侧损毁影响鸣曲音节基频和高频的稳定性

Figure 6 Changes in fundamental frequency and high frequency of syllables before and after right side nuclei lesion

$\mathrm{A}$ : 术前基频稳定, RA 被损后基频变化不明显, DM 被损后基频显著上 扬; B: 术前高频较高且稳定, RA 被损后高频显明下降, DM 被损后高 频再次下降。

A: fundamental frequency was steady before the operation, did not vary after RA was destroyed, but showed significant uptrend after DM lesion; B: before operation male birds had a steady and higher high frequency, which significantly descended after RA lesion and again after DM lesion.

的实验鸟需经灌流后取脑, 将脑切片后进行尼氏染 色，检验损毁位点，当组织学与行为学结果一致时 则判定为有效数据再进行分析统计。

\section{3 讨 论}

斑胸草雀鸣唱行为呈性别二态性, 雄鸟通过幼 龄期学习能产生复杂多变的习得性发声, 而雌鸟只 能发出非习得性的简单鸣叫, 与此对应的控制发声 的神经系统亦有明显的性别二态性, 雄鸟脑内的发 声核团明显大于雌岛(Bottjer et al, 1997; MacDougallShackleton \& Ball, 1999; Li \& Li, 1999)。神经解剖与 电生理实验也发现雌性脑内的核团间联系也较雄 性少很多(Fukushima \& Aoki, 2002)。雄鸟如不经过 学习教习曲或端脑功能缺陷也不能发出带有雄性 特征的声音。

\section{1 长鸣}

Vicario et al (2001)通过损毁双侧 RA 核团后分 析成年雄性斑胸草雀长鸣的变化及其对声音刺激 
的反应。损毁前, 雄鸟发出的是雄性特征的长鸣, 即有较高且稳定的基频、相对较短的时程并伴有快 速调频特性; 一些雄鸟对雌雄同类声音的刺激反应 差别较大, 雌鸟的声音能刺激其发出较多的长鸣。 而损毁后, 雄鸟声音发生变异, 出现了鸣叫时程延 长和快速调频特性的丧失。另外, 雄鸟对雌雄同类 声音的刺激也不再出现差别反应。我们损毁斑胸草 雀右侧 RA 后所得的声学结果与 Vicario et al 的报道 相似，共同支持习得性发声由前脑核团控制产生的 观点(Simpson \& Vicario, 1990); 但值得一提的是, 从本研究的结果得知, 损毁右侧 RA 后, 长鸣时域 和频域发生显著变化, 而损毁左侧 RA 对长鸣几乎 没有影响。鸣禽与人类相似, 其发声中枢存在侧别 优势, 多数鸣禽的发声呈左脑优势, 而斑胸草雀的 鸣叫中枢呈右脑优势 (Nottebohm \& Nottebohm, 1976; Li \& Qin, 2009), 所以, 我们认为是右侧高位 中枢指导习得性声音的产生。

除前脑高级中枢外，中脑的基本中枢对习得性 发声也有重要影响。过去认为, 中脑、延髓的发声 相关核团参与无性别差别的本能发声, 不参与复杂 的习得性发声。但实验表明, 刺激延髓的呼吸核团 也能造成类似刺激高级发声中枢所产生的发声干 扰。另外，对于中脑 DM 核团而言，它接受丘间核 复合体抑制性信号的输入, 是两性鸣禽均能发出长 鸣的关键结构, 雄鸟的 DM 接受来自同侧 RA 的突 触输入, 这一条路径已被证实为属于发声通路的一 部分(Ashmore et al, 2005), 所以, 雄鸟 DM 核团在 神经环路和功能上都有别于雌鸟的 DM 核团。我们 的研究表明, 损毁右侧 DM 后, 长鸣的某些声学参 数仍在发生变化, 说明 DM 核团参与习得性发声的 调节。

$R A$ 部分投射神经元与呼吸网络(由 DM、RAm、 PAm 核团组成)构成联系(Ashmore et al, 2005), DM 核团本身为呼吸网络的一部分, 由此可知呼吸控制 相关核团受高级中枢支配，当 RA 被损毁后，呼吸 网络失去高位调节而引起呼吸紊乱, 从而造成发声 时程延长, 如口吃一般。DM 核团既作为发声通路 中的一部分又作为呼吸网络的一部分, 它对发声与

\section{参考文献:}

Ashmore RC, Wild JM, Schmidt MF. 2005. Brainstem and forebrain contributions to the generation of learned motor behaviors for song. Journal of Neuroscience, 25(37): 8534-8543.
呼吸的影响至关重要, 它的缺失亦对发声时程造成 显著延长。实验表明, 刺激两性鸣禽的 DM 核团能 诱发出类似长距鸣叫的叫声, 诱发叫声的时程取决 于刺激的脉冲数。随刺激脉冲数增加, 叫声时程相 对较长, 反之则短。这说明在性成熟的两性鸣禽中, DM 核团中的神经系统可能控制着长距鸣叫的时程 (Fukushima \& Aoki, 2002)。

\section{2 鸣曲}

损毁右侧 RA 核团和 DM 核团后鸣曲出现变化, 与长鸣的变化不同。第一次损毁后，鸣曲立即发生 变化, 鸣曲中音节的频率变化类似于长鸣的频率变 化, 声音结构由复杂变简单, 调频特性消失, 音节 趋于恒频，声色暗哑没有起伏。术前音节的高低频 稳定, 能量带清晰, 高端频率可 $>20 \mathrm{kHz}$, 基低也 较为稳定, 第一次手术后( RA 核团的损毁), 音节结 构已出现上述频率特征的改变, 能量带界线不清, 高端频率出现显著下降, 只达到 $15 \mathrm{kHz}$, 单个音 节时程缩短，每个音节相似; 第二次手术后(DM 核 团的损毁), 语图中的音节更模糊, 高端再次有所下 降，降到 $13 \mathrm{kHz}$, 基频也开始不稳定, 出现飘移。

调控鸣曲变异的因素有很多, 前运动核团 HVC 和 RA 的神经元在产生鸣唱时同时激活, 支配 鸣管发声。社会情境对成年鸣曲的序列和音节可变 性也有调节作用(Sakata et al, 2008)。幼龄期前端脑 通路助于鸣曲的形成。正如我们所提到, 鸣曲是成 串的声音信号, 某一次的发声机会中, 鸣禽可能发 出一次长鸣或一首鸣曲，控声核团被损毁后，虽然 单个音节时程缩短了, 但整首鸣曲的时程可能延长, 音节数量可能增加, 这与呼吸失调是相关联的。另 外, 鸣曲的编码, 如序列及节奏等是 $\mathrm{HVC}$ 与脑干中 有神经折返回前脑的核团所共同作用而产生，所以， 我们推测不损及 $\mathrm{HVC}$ 和脑干的情况下, 鸣曲的改 变更多在于音节的改变。此外, 右侧实验组的鸣曲 较为重大的变化是高低端频率的缺失, 这个现象在 中脑核团被损后犹为突出, 这与刺激中脑 DM 时所 记录的发声相似，即基频升高，高频下降，所以， 我们推测中脑在调控音节的高低频中起着关键 作用。
Bottjer SW, Roselinsky H, Tran NB. 1997. Sex differences in neuropeptide b staining of song-control nuclei in zebra finch brains. Brain Behavior and Evolution, 50(5): 284-303. 
Brainard MS, Doupe AJ. 2002. What songbirds teach us about learning. Nature, 417(6886): 351-358.

Doupe AJ, Solis MM, Kimpo R, Boettiger CA. 2004. Cellular, circuit, and synaptic mechanisms in song learning. Annuals of the New York Academy of Sciences, 1016(1): 495-523.

Doupe AJ, Perkel DJ, Reiner A, Stern EA. 2005. Birdbrains could teach basal ganglia research a new song. Trends in Neurosciences, 28(7): 353-363.

Fukushima Y, Aoki K. 2002. Neural function of the mesencephalic dorsomedial nucleus (DM) on distance call production in Bengalese finches. Zoological Science, 19(4): 393-402.

Funabiki Y, Funabiki K. 2009. Factors limiting song acquisition in adult zebra finches. Developmental Neurobiology, 69(11): 752-759.

Gurney ME. 1980. Sexual Differentiation of Brain and Behavior in the Zebra Finch (Poephila Guttata): A Cellular Analysis. California: Caltech, 312 .

Kojima S, Aoki K. 2003. Intrinsic and synaptic properties of the dorsomedial nucleus of the intercollicular complex, an area known to be involved in distance call production in Bengalese finches. Brain Research, 966(1): 84-94.

Li DF, Li J. 1999. The hormonal regulation of vocal behavior in songbirds. Zoological Research, 20(1): 62-66. [李东风, 李杰. 1999. 鸣禽鸟发声行为 的激素调节. 动物学研究, 20(1): 62-66.]

Li DF, Geng H. 2005. The application of wavesurfer in birdsong analysis. Journal of Liaoning Normal University: Natural Science Edition, 28(4): 454-456. [李东风, 耿慧. 2005. 声谱分析软件 Wavesurfer 在鸟类鸣声分 析中的应用. 辽宁师范大学学报: 自然科学版, 28(4): 454-456.]

Li DF, Qin C. 2009. Spectrogram analysis of evoked call of DM nucleus in zebra finch (Taeniopygia guttata). Journal of Shangqiu Teacher College, 25(6): 1-6. [李东风, 秦川. 2009. 斑胸草雀中脑丘间复合体背内侧核诱 发鸣声的声谱分析. 商丘师范学院学报, 25(6): 1-6.]

Li DF, Zuo MX, Lan SC, Hu J. 1992. Sex differences of vocal control nuclei in the forebrain of songbirds. Acta Zoologica Sinica, 38(3): 298-304. [李东 风, 左明雪, 蓝书成, 胡建. 1992. 鸣而前脑发声控制核团的雌雄差别. 动物学报, 38(3): 298-304.]

MacDougall-Shackleton SA, Ball GF. 1999. Comparative studies of sex differences in the song-control system of songbirds. Trends in Neurosciences, 22(10): 432-436.

Nixdorf-Bergweiler BE, Bischof HJ. 2007. A Stereotaxic Atlas of the Brain of the Zebra Finch, Taeniopygia guttata, with Special Emphasis on Telencephalic Visual and Song System Nuclei in Transverse and Sagittal sections. Bethesda (MD): National Library of Medicine (US), National Center for Biotechnology Information.

Nottebohm F, Arnold AP. 1976. Sexual dimorphism in vocal control areas of the songbird brain. Science, 194(4261): 211-213.

Nottebohm F, Nottebohm ME. 1976. Left hypoglossal dominance in the control of canary and white-crowned sparrow song, Journal of Comparative Physiology, 108(2): 171-192.

Nottebohm F. 2005. The neural basis of birdsong. PLoS Biology, 3(5): 164-166.

Sakata JT, Hampton CM, Brainard MS. 2008. Social modulation of sequence and syllable variability in adult birdsong. Journal of Neurophysiology, 99(4): 1700-1711.

Simpson HB, Vicario DS. 1990. Brain pathways for learned and unlearned vocalizations differ in zebra finches. $J$ of Neurosciences, 10(5): 1541-1556.

Tchernichovski O, Nottebohm F, Ho CE, Pesaran B, Mitra PP. 2000. Procedure for an automated measurement of song similarity. Animal Behavior, 59(6): 1167-1176.

Troyer TW, Bottjer SW. 2001. Birdsong: models and mechanisms. Current Opinion in Neurobiology, 11(6): 721-726.

Vicario DS, Naqvi NH, Raksin JN. 2001. Behavioral discrimination of sexually dimorphic calls by male zebra finches requires an intact vocal motor pathway. J of Neurobiology, 47(2): 109-120. 